\section{Psychiatry and psychology}

\section{G189 AUDIT OF THE SPECIALIST ADHD CLINIC IN THANET REGION IN SOUTH EAST KENT}

S. Baneriee. School \& Child Health, East Kent Hospitals NHS Trust, Queens House, Queen Street, Ramsgate, Kent

Introduction: A Specialist ADHD Clinic is functioning in the Thanet region since April 1998.

Aims: This audit was done to evaluate the existing practice in this specialist ADHD Clinic against the NICE' and $\mathrm{SIGN}^{2}$ guidance standards.

Methods: A retrospective case note study of 50 children attending the Joint ADHD Clinic between October - December 2001.

Results: There were $46(92 \%)$ male children. The majority of the children were referred by school $(36 \%)$ and GPs $(30 \%)$. Two third of the children $(n=33)$ were school age at the time of the first referral. In 12 (24\%) children Conners' Rating Scales were used as an assessment tool. 10 children (82\%) were found to be positive both on Parents' and Teachers' Conners' Rating Scales. In cases of 45 children $(90 \%)$, letters were sent to school about the child's medication, whereas only in 13 cases $(26 \%)$ parents were given a copy of the letter. In $43(86 \%)$ the management plan was both behavioural management strategies and drugs. In $24(48 \%)$ cases there was no record of height and weight. There was no transitional plan for any of the 8 children who were over 14 years of age.

Conclusions: Although we offer a service, we did not meet all the standards as suggested in the SIGN and NICE guidelines. Parents and teachers should get a copy of each consultation. A joint care pathway with GPs for repeat prescriptions and monitoring needs to be devised and a transitional plan needs in place to meet the needs of young people leaving the education system at 16-19 yrs.

1. National Institute for Clinical Excellence. Technology Appraisal Guidance No.13. Guidance on the use of Methylphenidate for Attention Deficit/Hyperactivity Disorder (ADHD) in Childhood. London, October 2000.

2. Scottish Intercollegiate Guidelines Network. Attention Deficit and Hyperactive Disorders in Children and Young People: A National Clinical Guideline. SIGN, Executive Royal College of Physicians, Edinburgh, June 2001.

\section{G190 PHARMACOLOGIC PROPERTIES OF ATOMOXETINE: A NOVEL TREATMENT FOR ATTENTION DEFICIT/HYPERACTIVITY DISORDER}

S. Prasad, V. Harpin, F. Bymaster, J. Witcher, D. Michelson, W.J. Brown. Eli Lilly and Company Limited, Hampshire, UK

Background: Atomoxetine is a new, non-stimulant medication currently being studied for the treatment of ADHD.

Objectives: The purpose of this presentation is to review the pharmacologic properties of atomoxetine including chemical structure, proposed mechanism of action, and pharmacokinetics.

Methods: The pharmacologic properties of atomoxetine have been extensively studied and well characterized in animal and human models as part of the early clinical development process. Results from these studies will be summarized.

Results: Atomoxetine $\mathrm{HCl}$ is known chemically as benzenepropanamine, $\mathrm{N}$-methyl- $\gamma$ (2-methylphenoxy), hydrochloride, (-). Atomoxetine is a highly specific, presynaptic inhibitor of the norepinephrine transporter and has little affinity for other neurotransmitter receptor or transporter sites. This specificity provides evidence for the proposed mechanism of action and offers an explanation for the lack of some adverse events associated with currently available treatments for ADHD. Atomoxetine is rapidly absorbed following oral administration and is metabolized by the genetically polymorphic CYP2D6 enzyme pathway.

Conclusion: Atomoxetine is a novel treatment for ADHD with unique pharmacologic properties that distinguish it from currently available treatments for ADHD.
G191 ATOMOXETINE, A NEW NON-STIMULANT TREATMENT FOR ADHD: RESULTS OF AN INTERNATIONAL MULTI CENTRE OPEN LABEL TRIAL

V. Harpin, S. Prasad. Eli Lilly and Co Ltd, Hampshire, UK

Introduction: ADHD is a neurobiological disorder characterized by the core symptoms of impulsivity, hyperactivity and inattention. The prevalence of the disorder is estimated to be 4 to $6 \%$. Atomoxetine is a new, non-stimulant medication currently being studied as a treatment for ADHD. Atomoxetine is a highly specific inhibitor of the presynaptic norepinephrine transporter and has minimal affinity for other receptors or transporters.

Methods: More than 600 children and adolescents diagnosed with ADHD were treated with atomoxetine for a 10-week open-label treatment phase. The drug was given in the morning and evening, thus avoiding a school time dose. The study was conducted at 33 sites in the United Kingdom, France, Spain, Italy, Belgium, the Netherlands, Germany, Poland, Hungary, Sweden, Norway, Israel, South Africa and Australia. All patients had ADHD symptom severity at least 1.5 standard deviations above age and gender norms for their diagnostic subtype at the time of study entry as measured by the ADHD Rating Scale-IV-Parent Version:Investigator Scored (ADHD RS). Efficacy was measured by improvement in the ADHD RS and Clinical Global Impressions of Severity (CGI-ADHD-S). Improvement in family and social functioning was assessed with the Child Health Questionnaire (CHQ). Adverse events were assessed by open-ended questioning

Results: At endpoint, ADHD RS total scores decreased by an average of $56.7 \%(p<0.001)$. Similarly, the CGI-ADHD-S decreased by $51.9 \%$ ( $p<0.001)$. The $C H Q$ demonstrated improved scores. The most frequently occurring adverse events were gastrointestinal in origin and the discontinuation rate from the study attributed to adverse events was $3.8 \%$.

Conclusions: Atomoxetine appears to be an efficacious and welltolerated treatment in the treatment of ADHD symptoms in children and adolescents.

\section{G192 BABY EXPRESS: HELPING PARENTS TO UNDERSTAND THEIR NEW INFANT}

T. Waterston, B. Keane, H. McConachie, E. Towner, M. Cook. Universities of Newcastle upon Tyne and Northumbria

There is evidence both from the current epidemic of emotional and behavioural problems and the comments of parents themselves, that little help is available for new parents to understand their infant's emotional development. We studied the effects of an age-paced newsletter sent to the home every month over the first six months, on new parents in an Education Action Zone in a northern city.

The newsletter was locally edited and based in a US model, and focused on emotional development, play, and common problems with a special emphasis on the infant's perspective. Copies were sent to 100 mothers and interviews were carried out in the home. At the end of the period a parent hassle scale was applied to 34 mothers in the study group and to 32 controls.

60 mothers were interviewed over the six month period. Newsletters were delivered effectively by post, with $97 \%$ of those interviewed reading all or part of the content with good recall of topics. Mothers who had received and read the parenting newsletter liked the content very much, especially the section explaining the perspective of the infant. Many reported changes in parenting behaviour particularly with regards to response to crying and interacting with their baby through play. Typical comments were:

The bit on the games, that's where I got the idea of the game like opening my eyes and that 'cos I didn't know about it before and we started playing games like that and it's brilliant the way he responds

I like the bits about when the baby cries and how you should always go straight to them. I always pick her up and think you can't spoil a baby 'cos he says 'you're spoiling her' and I say 'you can't spoil her, if you read me leaflet (Baby Express) you'll see'

Parenting stress tended to be lower in the intervention group than controls.

An aged-paced parenting newsletter is an acceptable and popular format for supporting parenting of young infants. A longer study is underway using the Mind-Mindedness scale, which investigates the extent to which a parent sees her infant as an independent and autonomous person. 


\section{G193 FAMILY STRESS AND MATERNAL DEPRESSION FOLLOWING ADMISSION TO HOSPITAL FOR TRAUMATIC BRAIN INJURY}

A.L. Curran, H. Miller, R. McCarter, P.M. Sharples and the Kids Head Injury Study Group. Bristol

Introduction: Traumatic brain injury (TBI) is a major cause of admission to hospital in childhood, but few data exist concerning the impact of such an admission on family or maternal emotional health.

Aims: To determine the impact on the family and mother of hospitalisation of a child for TBI one month after admission.

Methods: Longitudinal prospective follow up study of a cohort of children admitted to hospital for TBI compared to non-injured control children. The Glasgow Coma Score (GCS) on hospital admission was used to divide TBI children into severe (GCS 3-8), moderate (GCS 9-12) and mild (GCS 13-15) categories. The Family Burden of Injury Inventory (FBII) was used to measure family stress. The Beck's Depression Inventory-II (BDI-II) was used to measure maternal emotional health.

Results: $53 \mathrm{TBI}$ children and 34 controls were recruited. There was no significant difference in age between TBI (mean 8.7 yrs; SEM 0.86 ) and control subjects (mean 9.3 yrs; SEM 0.97) $(p=0.62)$. The proportion of families showing evidence of significant stress (FBII score > 9) was significantly higher in TBI children, being $83 \%$ in the severe/ moderate TBI group, $50 \%$ mild TBI group and $0 \%$ in the control group $(p=0.0001)$. The proportion of mothers showing evidence of clinically significant depression (BDI-II score > 9) was also significantly higher in TBI children, being $55 \%$ in the severe/moderate TBI group, $28 \%$ in mild $\mathrm{TBI}$ group and $14 \%$ in the control group. Mothers of severe/moderate TBI subjects ( $n=20$ ) had significantly higher $B D I-1 \mid$ scores (mean 12.9, SEM 2.55) than mothers of control children (mean 3.8; SEM 1.08) ( $p=0.0007)$. There was a significant correlation between BDI-II scores in the mothers and FBII scores in the family $(p=0.0001)$

Conclusion: Admission to hospital with TBI in a child is associated with significant levels of maternal depression and family stress at one month. Follow up programmes for TBI children should include early psychological screening and support services for mothers and families.

\section{G194 BEHAVIOURAL AND EMOTIONAL FUNCTIONING OF CHILDREN WITH CANCER IN THE NINE MONTHS FOLLOWING DIAGNOSIS}

H. Kondryn, J. Hill, E. Mackie, O.B. Eden, K. Bull, R. Robinson, R. Goldwyn. Royal Manchester Children's Hospital; Royal Liverpool Children's Hospital; Southampton General Hospital

Aims: This study was designed to examine (1) child functioning in the nine months following cancer diagnosis and (2) post-diagnosis functioning in relation to estimates of pre-diagnosis functioning.

Method: Using a prospective design, parents and teachers of young children newly diagnosed with cancer $(n=83)$, completed the Child Behaviour Checklist and Teacher Report Form respectively. A well-matched control group of healthy school children $(n=80)$ was used.

Results: Cancer children and controls did not differ on any of the indices of externalising behaviours. No significant differences were found between the combined parent/teacher estimates of the cancer group prior to the illness on the anxious/depressed and withdrawal scores and the control group. The anxious/depressed and withdrawal scores of the children with cancer six weeks post-diagnosis were significantly higher than their pre-diagnosis scores. At nine months the cancer group had higher anxious/depressed scores than controls $(p=0.04)$ and significantly higher withdrawal scores $(p=0.018)$. Compared to controls there was also evidence of greater within cancer subject change in both anxious/depressed scores (chi-square = $10.57, p=0.001$ ) and withdrawal scores (chi-square $=9.72, p=$ 0.002)

Conclusion: Compared with well-matched controls, the cancer group exhibited increased withdrawal and symptoms of anxiety/depression. Furthermore, there is evidence that cancer children had a substantial increase in anxious/depressed symptoms and social withdrawal. This may mean that these are vulnerable children who do not yet show marked difficulties, but who nevertheless have started on a trajectory towards persistent and substantial social withdrawal. 